A class of $2 n$ th-order differential operator with eigenparameter-dependent boundary and transmission conditions

\author{
Xin-Yan Zhang and Jiong Sun
}




\title{
A CLASS OF $2 n$ TH-ORDER DIFFERENTIAL OPERATOR WITH EIGENPARAMETER-DEPENDENT BOUNDARY AND TRANSMISSION CONDITIONS
}

\author{
XIN-YAN ZHANG AND JIONG SUN
}

Received 19 March, 2012

\begin{abstract}
In this paper, we investigate a class of $2 n$ th-order regular differential operator with eigenparameter-dependent boundary conditions and transmission conditions at an interior discontinuous point. By constructing a new linear operator $A$ associated with the problem, we prove that the operator $A$ is self-adjoint in a suitable Hilbert space $H$, and the eigenvalues of the problem coincide with those of $A$. In terms of basic solutions of differential equation, we show that the eigenvalues of this problem coincide with the zeros of the entire function $\operatorname{det} \Phi(1, \lambda)$, and obtain that the operator $A$ has only point spectrum.
\end{abstract}

2000 Mathematics Subject Classification: 47E05; 34L10; 34B05

Keywords: $2 n$ th-order differential operator, eigenparameter-dependent boundary conditions, transmission conditions, eigenvalues, point spectrum

\section{INTRODUCTION}

The Sturm-Liouville theory is one of the most actual and extensively developing fields of theoretical and applied mathematics. In recent years, highly important results in this field have been obtained for the case when the eigenparameter appears not only in the differential equation but also in the boundary conditions. The literature on such results is voluminous and we refer to [1,2,4,5,7, 8, 11, 13, 14]. While the general theory and methods of such second-order boundary value problems are highly developed, very little is known about the general character of the higher order problems.

While dealing with interior discontinuousness, some boundary value problems with transmission conditions arise in heat and mass transfer, vibrating string problems, diffraction problems and various physical transfer problems [3, 6,9]. Also, some problems with transmission conditions which arise in mechanics [12]. In this

The research of the first and second authors is supported by the National Nature Science Foundation of China, Grant No.10861008, 11161030. ”211 Project” innovative talents training program of Inner Mongolia University, Grant No. 2-1.2.1-132, and the general project of Inner Mongolia University of Finiance and Economics. 
paper we shall investigate a class of $2 n$ th-order boundary value problems with eigenparameter boundary conditions and transmission conditions at an interior discontinuous point. The goal of this study is to extend some results of the standard S-L problems to the higher order discontinuous case.

We consider a $2 n$ th-order differential operator with eigenparameter boundary conditions and transmission conditions, and define a new linear operator $A$ associated with the problem in a suitable Hilbert space $H$, and prove the operator $A$ is selfadjoint, the eigenvalues of the problem coincide with those of $A$. We construct basic solutions, and show that the eigenvalues of this problem coincide with the zeros of the entire function $\operatorname{det} \Phi(1, \lambda)$, and get that the operator $A$ has only point spectrum.

This paper is organized as follows. In Section 1, we introduce the problem considered, a formulae of the operator $A$ in the suitable Hilbert space $H$ is constructed. In Section 2, we prove the operator $A$ is self-adjoint. Basic solutions are achieved in Section 3, and show that the eigenvalues of this problem coincide with the zeros of the entire function $\operatorname{det} \Phi(1, \lambda)$. In Section 4 , we prove that the operator $A$ has only point spectrum.

In this paper, we investigate a discontinuous boundary value problem which consists of the differential equation

$$
l u:=-\left(p(x) u^{(n)}\right)^{(n)}+q(x) u=\lambda u, x \in J,
$$

where $J=[-1,0) \cup(0,1], p(x)=p_{1}^{2}$ for $x \in[-1,0), p(x)=p_{2}^{2}$ for $x \in(0,1], p_{1}, p_{2}$ are nonzero real numbers; $q(x) \in L^{1}(J, R), \lambda \in \mathbb{C}$ is the so-called eigenparameter; with boundary conditions

$$
l_{i} u:=a_{i} u^{(i-1)}(-1)+a_{2 n+1-i} u^{(2 n-i)}(-1)=0, i=1,2, \cdots, n,
$$

the eigenparameter-dependent boundary conditions

$$
\begin{aligned}
l_{n+i} u & :=\lambda\left(b_{i}^{\prime} u^{(i-1)}(1)+b_{2 n+1-i}^{\prime} u^{(2 n-i)}(1)\right) \\
& +b_{i} u^{(i-1)}(1)+b_{2 n+1-i} u^{(2 n-i)}(1)=0, i=1,2, \cdots, n,
\end{aligned}
$$

and transmission conditions at the interior discontinuous point

$$
l_{2 n+i} u:=u^{(i-1)}(0+)-\sum_{j=1}^{2 n} c_{i j} u^{(j-1)}(0-)=0, i=1,2, \cdots, 2 n,
$$

where $a_{i}, b_{i}, b_{i}^{\prime}(i=1,2, \cdots, 2 n)$ are real numbers, $C=\left(c_{i j}\right)$ is $2 n \times 2 n$ real matrix, we assume that

$$
\begin{gathered}
a_{n+i} \neq 0(i=1,2, \cdots, n), \quad \operatorname{det} C=\rho^{n}, \rho>0, \quad C^{T} Q C=\rho Q, \\
\theta_{i}=\left|\begin{array}{cc}
b_{i}^{\prime} & b_{2 n+1-i}^{\prime} \\
b_{i} & b_{2 n+1-i}
\end{array}\right|>0, i=1,2, \cdots, n,
\end{gathered}
$$


where $Q$ is $2 n \times 2 n$ real matrix as follows

$$
Q=\left(\begin{array}{lllll} 
& & & & 1 \\
& & \ldots & & \\
& & & & \\
-1 & & & & \\
& & &
\end{array}\right)
$$

Let

$$
C_{u}(x)=\left(u(x), u^{\prime}(x), \cdots, u^{(2 n-1)}(x)\right)^{T} .
$$

In order to investigate the problem (1.1)-(1.4), we define a new inner product in $L^{2}(J)$ as

$$
\langle f, g\rangle_{1}=\frac{1}{p_{1}^{2}} \int_{-1}^{0} f_{1}(x) \overline{g_{1}(x)} d x+\frac{1}{p_{2}^{2} \rho} \int_{0}^{1} f_{2}(x) \overline{g_{2}(x)} d x, \forall f, g \in L^{2}(J),
$$

where $f_{1}(x)=\left.f(x)\right|_{[-1,0)}, f_{2}(x)=\left.f(x)\right|_{(0,1]}$. It is easy to verify that $H_{1}=\left(L^{2}(J),\langle\cdot, \cdot\rangle_{1}\right)$ is a Hilbert space.

\section{The Self-adjointness of Operator $A$}

In this section, we introduce the special inner product in the Hilbert space $H=$ $H_{1} \oplus \mathbb{C}^{n}$, where $H_{1}=\left(L^{2}(J),\langle\cdot, \cdot\rangle_{1}\right), \mathbb{C}$ denotes the set of complex numbers. A symmetric linear operator $A$ is defined on this Hilbert space such that problem (1.1)(1.4) can be considered as the eigenvalue problem of this operator. Here, we prove that the operator $A$ is self-adjoint, not only symmetric.

We define an inner product in $H$ by

$$
\begin{gathered}
\langle F, G\rangle=\langle f, g\rangle_{1}+\frac{1}{\rho} \sum_{i=1}^{n}(-1)^{i} \frac{1}{\theta_{i}} h_{i} \bar{k}_{i}, \\
\forall f, g \in H_{1}, h_{i}, k_{i} \in \mathbb{C}, i=1,2, \cdots, n,
\end{gathered}
$$

for

$$
F=\left(f, h_{1}, h_{2}, \cdots, h_{n}\right), G=\left(g, k_{1}, k_{2}, \cdots, k_{n}\right) \in H .
$$

In the Hilbert space $H$, we consider the operator $A$ which is defined by

$$
\begin{gathered}
D(A)=\left\{\left(f, h_{1}, h_{2}, \cdots, h_{n}\right) \in H \mid f_{1}^{(i-1)} \in A C_{l o c}((-1,0)),\right. \\
f_{2}^{(i-1)} \in A C_{l o c}((0,1)), i=1,2, \cdots, 2 n, l f \in H_{1}, \\
l_{j} f=0, j=1,2, \cdots, n, C_{f}(0+)=C \cdot C_{f}(0-), \\
\left.h_{i}=b_{i}^{\prime} f^{(i-1)}(1)+b_{2 n+1-i}^{\prime} f^{(2 n-i)}(1), i=1,2, \cdots, n\right\}, \\
A F=\left(l f,-\left(b_{1} f(1)+b_{2 n} f^{(2 n-1)}(1)\right),-\left(b_{2} f^{\prime}(1)+b_{2 n-1} f^{(2 n-2)}(1)\right),\right. \\
\left.\cdots,-\left(b_{n} f^{(n-1)}(1)+b_{n+1} f^{(n)}(1)\right)\right),
\end{gathered}
$$




$$
\begin{array}{r}
F=\left(f, b_{1}^{\prime} f(1)+b_{2 n}^{\prime} f^{(2 n-1)}(1), b_{2}^{\prime} f^{\prime}(1)+b_{2 n-1}^{\prime} f^{(2 n-2)}(1),\right. \\
\left.\cdots, b_{n}^{\prime} f^{(n-1)}+b_{n+1}^{\prime} f^{(n)}(1)\right) \in D(A) .
\end{array}
$$

For convenience, $\forall F=\left(f, h_{1}, h_{2}, \cdots, h_{n}\right) \in D(A)$, let

$$
\begin{aligned}
& M_{i}(f)=b_{i} f^{(i-1)}(1)+b_{2 n+1-i} f^{(2 n-i)}(1), i=1,2, \cdots, n, \\
& M_{i}^{\prime}(f)=b_{i}^{\prime} f^{(i-1)}(1)+b_{2 n+1-i}^{\prime} f^{(2 n-i)}(1), i=1,2, \cdots, n,
\end{aligned}
$$

so by (1.1), (1.3) we have

$$
\begin{aligned}
A F & =\left(l f,-M_{1}(f),-M_{2}(f), \cdots,-M_{n}(f)\right) \\
& =\left(\lambda f, \lambda M_{1}^{\prime}(f), \lambda M_{2}^{\prime}(f), \cdots, \lambda M_{n}^{\prime}(f)\right)=\lambda F .
\end{aligned}
$$

Now we can rewrite the considered problem (1.1)-(1.4) in the operator form $A F=$ $\lambda F$.

From (1.6), (2.3) and (2.4), by direct calculation we obtain the following Lemma.

Lemma 1. If the functions $f(x)$ and $g(x)$ are differential on the interval $[0,1]$, then

$$
W(f, \bar{g} ; 1)=\sum_{i=1}^{n}(-1)^{i} \frac{1}{\theta_{i}} M_{i}(f) M_{i}^{\prime}(\bar{g})-\sum_{i=1}^{n}(-1)^{i} \frac{1}{\theta_{i}} M_{i}^{\prime}(f) M_{i}(\bar{g}),
$$

where

$$
\begin{aligned}
& W(f, g ; x) \\
& \quad=f(x) g^{(2 n-1)}(x)-f^{\prime}(x) g^{(2 n-2)}(x)+\cdots+(-1)^{n-1} f^{(n-1)}(x) g^{(n)}(x) \\
& \quad+(-1)^{n} f^{(n)}(x) g^{(n-1)}(x)+\cdots+f^{(2 n-2)}(x) g^{\prime}(x)-f^{(2 n-1)}(x) g(x) \\
& =\sum_{i=1}^{n}(-1)^{i+1}\left(f^{(i-1)}(x) g^{(2 n-i)}(x)-f^{(2 n-i)}(x) g^{(i-1)}(x)\right)=C_{f}^{T}(x) Q C_{g}(x) .
\end{aligned}
$$

Proof. By (1.6), (2.3) and (2.4), we have

$$
\begin{gathered}
\sum_{i=1}^{n}(-1)^{i} \frac{1}{\theta_{i}} M_{i}(f) M_{i}^{\prime}(\bar{g})-\sum_{i=1}^{n}(-1)^{i} \frac{1}{\theta_{i}} M_{i}^{\prime}(f) M_{i}(\bar{g}) \\
=\sum_{i=1}^{n}(-1)^{i} \\
\frac{1}{\theta_{i}}\left(\left(b_{i} f^{(i-1)}(1)+b_{2 n+1-i} f^{(2 n-i)}(1)\right)\left(b_{i}^{\prime} \bar{g}^{(i-1)}(1)+b_{2 n+1-i}^{\prime} \bar{g}^{(2 n-i)}(1)\right)\right. \\
\left.-\left(b_{i}^{\prime} f^{(i-1)}(1)+b_{2 n+1-i}^{\prime} f^{(2 n-i)}(1)\right)\left(b_{i} \bar{g}^{(i-1)}(1)+b_{2 n+1-i} \bar{g}^{(2 n-i)}(1)\right)\right) \\
=\sum_{i=1}^{n}(-1)^{i}
\end{gathered}
$$




$$
\begin{aligned}
& \frac{1}{\theta_{i}}\left(b_{i} b_{2 n+1-i}^{\prime}-b_{i}^{\prime} b_{2 n+1-i}\right)\left(f^{(i-1)}(1) \bar{g}^{(2 n-i)}(1)-f^{(2 n-i)}(1) \bar{g}^{(i-1)}(1)\right) \\
= & \sum_{i=1}^{n}(-1)^{i+1}\left(f^{(i-1)}(1) \bar{g}^{(2 n-i)}(1)-f^{(2 n-i)}(1) \bar{g}^{(i-1)}(1)\right)=W(f, \bar{g} ; 1) .
\end{aligned}
$$

Lemma 2. The eigenvalues of the problem (1.1)-(1.4) coincide with those of operator $A$, and its eigenfunctions are the first component of corresponding eigenfunctions of the operator $A$.

Lemma 3. The domain $D(A)$ of the operator $A$ is dense in $H$.

Proof. Suppose that there is an element $F=\left(f, h_{1}, h_{2}, \cdots, h_{n}\right) \in H$, which is orthogonal to all $U=\left(u, M_{1}^{\prime}(u), M_{2}^{\prime}(u), \cdots, M_{n}^{\prime}(u)\right) \in D(A)$ in Hilbert space $H$, i.e.

$$
\begin{aligned}
\langle F, U\rangle= & \frac{1}{p_{1}^{2}} \int_{-1}^{0} f_{1}(x) \overline{u_{1}(x)} d x+\frac{1}{p_{2}^{2} \rho} \int_{0}^{1} f_{2}(x) \overline{u_{2}(x)} d x+ \\
& \frac{1}{\rho} \sum_{i=1}^{n}(-1)^{i} \frac{1}{\theta_{i}} h_{i} M_{i}^{\prime}(\bar{u}) .
\end{aligned}
$$

Let $\widetilde{C_{0}^{\infty}}$ be the set of all functions defined on $[-1,0) \cup(0,1]$ such that

$$
\phi(x)= \begin{cases}\varphi_{1}(x), & x \in[-1,0), \\ \varphi_{2}(x), & x \in(0,1],\end{cases}
$$

for $\varphi_{1}(x) \in C_{0}^{\infty}[-1,0), \varphi_{2}(x) \in C_{0}^{\infty}(0,1]$. As usual, from the well-known fact that $C_{0}^{\infty}(a, b)$ is dense in the Hilbert space $L^{2}(a, b)$ [14, p96], it follows that the set $\widetilde{C_{0}^{\infty}}$ is dense in the Hilbert space $H_{1}$. Since $\widetilde{C_{0}^{\infty}} \oplus 0^{n} \subset D(A)(0 \in \mathbb{C})$ and $U=$ $(u(x), 0, \cdots, 0) \in \widetilde{C_{0}^{\infty}} \oplus 0^{n}$ is orthogonal to $F$, i.e.

$$
\langle F, U\rangle=\frac{1}{p_{1}^{2}} \int_{-1}^{0} f_{1}(x) \overline{u_{1}(x)} d x+\frac{1}{p_{2}^{2} \rho} \int_{0}^{1} f_{2}(x) \overline{u_{2}(x)} d x=\langle f, u\rangle_{1}=0 .
$$

Therefore, (2.6) means that $f(x)$ is orthogonal to the subspace $\widetilde{C_{0}^{\infty}}$ which is dense everywhere in the Hilbert space $H_{1}$, so $f(x)$ is null element of $H_{1}$, putting $f(x)=0$ in (2.5), we have

$$
\frac{1}{\rho} \sum_{i=1}^{n}(-1)^{i} \frac{1}{\theta_{i}} h_{i} M_{i}^{\prime}(\bar{u})=0,
$$

for all $u \in H_{1}$, such that $U \in D(A)$. So for all $G_{1}=\left(g(x), M_{1}^{\prime}(g), 0, \cdots, 0\right) \in D(A)$,

$$
\left\langle F, G_{1}\right\rangle=\langle f, g\rangle_{1}+\frac{1}{\rho \theta_{1}} h_{1} M_{1}^{\prime}(\bar{g})=0,
$$


since $M_{1}^{\prime}(g)$ can be chosen arbitrarily, so $h_{1}=0$. Similarly $h_{2}=h_{3}=\cdots=h_{n}=$ 0 . Hence $F=(0,0, \cdots, 0)$ is the null element of the Hilbert space $H$. Thus, the orthogonal complement of $D(A)$ consists of only the null element, and therefore is dense in the Hilbert space $H$.

Theorem 1. The operator $A$ is self-adjoint in $H$.

Proof. Let $F$ and $G$ be arbitrary elements of $D(A)$, by partial integrations we obtain

$$
\begin{aligned}
\langle A F, G\rangle= & \langle F, A G\rangle+W(f, \bar{g} ; 0-)-W(f, \bar{g} ;-1)+\frac{1}{\rho} W(f, \bar{g} ; 1) \\
& -\frac{1}{\rho} W(f, \bar{g} ; 0+)+\frac{1}{\rho} \sum_{i=1}^{n}(-1)^{i} \frac{1}{\theta_{i}} M_{i}^{\prime}(f) M_{i}(\bar{g}) \\
& -\frac{1}{\rho} \sum_{i=1}^{n}(-1)^{i} \frac{1}{\theta_{i}} M_{i}(f) M_{i}^{\prime}(\bar{g}) .
\end{aligned}
$$

Since $f$ and $g$ satisfy the boundary conditions (1.2), and $a_{n+i} \neq 0(i=1,2, \cdots, n)$, it follows that

$$
W(f, \bar{g} ;-1)=0 .
$$

From the transmission conditions (1.4) we get

$$
\begin{aligned}
W(f, \bar{g} ; 0+) & =C_{f}^{T}(0+) Q C_{\bar{g}}(0+)=C_{f}^{T}(0-) C^{T} Q C C_{\bar{g}}(0-) \\
& =\rho C_{f}^{T}(0-) Q C_{\bar{g}}(0-)=\rho W(f, \bar{g} ; 0-) .
\end{aligned}
$$

Further, putting (2.8) and (2.9) into (2.7), and applying Lemma 1, we get the required equality

$$
\langle A F, G\rangle=\langle F, A G\rangle(F, G \in D(A)),
$$

so $A$ is symmetric.

It remains to show that if $\langle A F, W\rangle=\langle F, U\rangle$ for all $F=\left(f(x), M_{1}^{\prime}(f), M_{2}^{\prime}(f)\right.$, $\left.\cdots, M_{n}^{\prime}(f)\right) \in D(A)$ then $W \in D(A)$ and $A W=U$. Where $W=\left(w(x), h_{1}, h_{2}\right.$, $\left.\cdots, h_{n}\right), U=\left(u(x), k_{1}, k_{2}, \cdots, k_{n}\right)$, i.e.

(1) $w_{1}^{(i-1)} \in A C_{l o c}((-1,0)), w_{2}^{(i-1)} \in A C_{l o c}((0,1)), i=1,2, \cdots, 2 n$, and $l w \in H_{1}$

(2) $h_{i}=M_{i}^{\prime}(w)=b_{i}^{\prime} w^{(i-1)}(1)+b_{2 n+1-i}^{\prime} w^{(2 n-i)}(1), i=1,2, \cdots, n$;

(3) $l_{i} w=a_{i} w^{(i-1)}(-1)+a_{2 n+1-i} w^{(2 n-i)}(-1)=0, i=1,2, \cdots, n$;

(4) $C_{w}(0+)=C \cdot C_{w}(0-)$;

(5) $u(x)=l w$

(6) $k_{i}=-M_{i}(w)=-\left(b_{i} w^{(i-1)}(1)+b_{2 n+1-i} w^{(2 n-i)}(1)\right), i=1,2, \cdots, n$. 
For an arbitrary $F \in \widetilde{\mathbb{C}_{0}^{\infty}} \oplus 0^{n} \subset D(A)$, from $\langle A F, W\rangle=\langle F, U\rangle$, we have

$$
\frac{1}{p_{1}^{2}} \int_{-1}^{0}(l f) \bar{w} d x+\frac{1}{p_{2}^{2} \rho} \int_{0}^{1}(l f) \bar{w} d x=\frac{1}{p_{1}^{2}} \int_{-1}^{0} f \bar{u} d x+\frac{1}{p_{2}^{2} \rho} \int_{0}^{1} f \bar{u} d x,
$$

that is $\langle l f, w\rangle_{1}=\langle f, u\rangle_{1}$. According to normal Sturm-Liouville theory, (1) and (5) hold. By (5), the equation $\langle A F, W\rangle=\langle F, U\rangle, \forall F \in D(A)$ becomes

$$
\begin{aligned}
& \frac{1}{p_{1}^{2}} \int_{-1}^{0}(l f) \bar{w} d x+\frac{1}{p_{2}^{2} \rho} \int_{0}^{1}(l f) \bar{w} d x-\frac{1}{\rho} \sum_{i=1}^{n}(-1)^{i} M_{i}(f) \bar{h}_{i} \\
& =\frac{1}{p_{1}^{2}} \int_{-1}^{0} f(l \bar{w}) d x+\frac{1}{p_{2}^{2} \rho} \int_{0}^{1} f(l \bar{w}) d x+\frac{1}{\rho} \sum_{i=1}^{n}(-1)^{i} M_{i}^{\prime}(f) \bar{k}_{i},
\end{aligned}
$$

so

$$
\langle l f, w\rangle_{1}=\langle f, l w\rangle_{1}+\frac{1}{\rho} \sum_{i=1}^{n}(-1)^{i} \frac{1}{\theta_{i}} M_{i}(f) \bar{h}_{i}+\frac{1}{\rho} \sum_{i=1}^{n}(-1)^{i} \frac{1}{\theta_{i}} M_{i}^{\prime}(f) \overline{k_{i}} .
$$

However

$$
\begin{aligned}
& \langle l f, w\rangle_{1}=\frac{1}{p_{1}^{2}} \int_{-1}^{0}(l f) \bar{w} d x+\frac{1}{p_{2}^{2} \rho} \int_{0}^{1}(l f) \bar{w} d x \\
& =\frac{1}{p_{1}^{2}} \int_{-1}^{0}\left(-p_{1}^{2} f^{(2 n)}+q(x) f\right) \bar{w} d x+\frac{1}{p_{2}^{2} \rho} \int_{0}^{1}\left(-p_{2}^{2} f^{(2 n)}+q(x) f\right) \bar{w} d x \\
& =\frac{1}{p_{1}^{2}} \int_{-1}^{0} f(l \bar{w}) d x+\frac{1}{p_{2}^{2} \rho} \int_{0}^{1} f(l \bar{w}) d x+W(f, \bar{w} ; 0-)-W(f, \bar{w} ;-1) \\
& \quad+\frac{1}{\rho} W(f, \bar{w} ; 1)-\frac{1}{\rho} W(f, \bar{w} ; 0+) \\
& =\langle f, l w\rangle_{1}+W(f, \bar{w} ; 0-)-W(f, \bar{w} ;-1)+\frac{1}{\rho} W(f, \bar{w} ; 1)-\frac{1}{\rho} W(f, \bar{w} ; 0+) .
\end{aligned}
$$

Hence,

$$
\begin{array}{r}
\langle l f, w\rangle_{1}=\langle f, l w\rangle_{1}+W(f, \bar{w}, 0-)-W(f, \bar{w},-1) \\
+\frac{1}{\rho} W(f, \bar{w}, 1)-\frac{1}{\rho} W(f, \bar{w}, 0+),
\end{array}
$$

so

$$
\begin{array}{r}
\frac{1}{\rho} \sum_{i=1}^{n}(-1)^{i} \frac{1}{\theta_{i}} M_{i}(f) \bar{h}_{i}+\frac{1}{\rho} \sum_{i=1}^{n}(-1)^{i} \frac{1}{\theta_{i}} M_{i}^{\prime}(f) \overline{k_{i}} \\
=W(f, \bar{w}, 0-)-W(f, \bar{w},-1)+\frac{1}{\rho} W(f, \bar{w}, 1)-\frac{1}{\rho} W(f, \bar{w}, 0+) .
\end{array}
$$


By Naimark Patching Lemma [10], there is an $F \in D(A)$, such that

$$
\begin{gathered}
f^{(i-1)}(-1)=f^{(i-1)}(0-)=f^{(i-1)}(0+)=0, i=1,2, \cdots, 2 n, \\
f(1)=-b_{2 n}^{\prime}, f^{(i)}(1)=0, i=1,2, \cdots, 2 n-2, f^{(2 n-1)}(1)=b_{1}^{\prime},
\end{gathered}
$$

For such an $F, M_{i}^{\prime}(f)=0, i=1,2, \cdots, n, M_{1}(f)=\theta_{1}, M_{i}(f)=0, i=2, \cdots, 2 n$. Then from (2.10) we have $h_{1}=M_{1}^{\prime}(w)$. Similarly, we can prove $h_{i}=M_{i}^{\prime}(w), i=$ $2, \cdots, n$. So the equalities (2) hold.

Similarly, we can prove (6).

Next choose $F \in D(A)$, such that

$$
\begin{array}{r}
f^{(i-1)}(0+)=f^{(i-1)}(0-)=f^{(i-1)}(1)=0, i=1,2, \cdots, 2 n, f(-1)=a_{2 n}, \\
f^{(i-1)}(-1)=0, i=2, \cdots, 2 n-1, f^{(2 n-1)}(-1)=-a_{1},
\end{array}
$$

thus $M_{i}(f)=M_{i}^{\prime}(f)=0, i=1,2, \cdots, n$. Then from (2.10) we have

$$
W(f, \bar{w},-1)=a_{1} \bar{w}(-1)+a_{2 n} \bar{w}^{(2 n-1)}(-1)=0,
$$

that is $l_{1} \bar{w}=0$, so $l_{1} w=0$. Similarly, we can prove $l_{i} w=0, i=2, \cdots, n$. So the equalities (3) hold.

Next choose $F \in D(A)$, such that

$$
\begin{gathered}
f^{(i-1)}(-1)=f^{(i-1)}(1)=0, i=1,2, \cdots, 2 n, f^{(i-1)}(0+)=0, i=1,2, \cdots, \\
2 n-1, f^{(2 n-1)}(0+)=\rho, f^{(i-1)}(0-)=(-1)^{i} c_{1,2 n+1-i}, i=1,2, \cdots, 2 n,
\end{gathered}
$$

thus $M_{i}(f)=M_{i}^{\prime}(f)=0, i=1,2, \cdots, n$. Then from (2.10) we have

$$
W(f, \bar{w} ; 0+)=\rho W(f, \bar{w} ; 0-) .
$$

However, $C=\left(c_{i j}\right)$ is $2 n \times 2 n$ real matrix, then $w(0+)=\sum_{i=1}^{2 n} c_{1 i} w^{(i-1)}(0-)$. Using the same method, we can prove

$$
w^{(k-1)}(0+)=\sum_{i=1}^{2 n} c_{k i} w^{(i-1)}(0-), k=2,3, \cdots, 2 n,
$$

that is $C_{w}(0+)=C \cdot C_{w}(0-)$. So the equality (4) holds.

From the above synthesis proof, we can know $A$ is a self-adjoint operator.

From the properties of self-adjoint operators, we have

Corollary 1. All the eigenvalues of the considered problem (1.1)-(1.4) are real.

Corollary 2. Let $\lambda_{1}$ and $\lambda_{2}$ be two different eigenvalues of the problem (1.1)-(1.4), then the corresponding eigenfunctions $f(x)$ and $g(x)$ are orthogonal in the sense of

$$
\frac{1}{p_{1}^{2}} \int_{-1}^{0} f \bar{g} d x+\frac{1}{p_{2}^{2} \rho} \int_{0}^{1} f \bar{g} d x+\frac{1}{\rho} \sum_{i=1}^{n}(-1)^{i} \frac{1}{\theta_{i}}
$$




$$
\left(b_{i}^{\prime} f^{(i-1)}(1)+b_{2 n+1-i}^{\prime} f^{(2 n-i)}(1)\right)\left(b_{i}^{\prime} \bar{g}^{(i-1)}(1)+b_{2 n+1-i}^{\prime} \bar{g}^{(2 n-i)}(1)\right)=0 .
$$

Consequently, the eigenfunctions of the problem (1.1)-(1.4) corresponding to the different eigenvalues are not orthogonal in the usual sense in the Hilbert space $H$.

\section{Construction of Basic Solutions}

In terms of existence and uniqueness theorem in ordinary differential equation theory, we shall define two group basic solutions $\phi_{1}(x, \lambda), \phi_{2}(x, \lambda), \cdots$, $\phi_{n}(x, \lambda)$ and $\chi_{1}(x, \lambda), \chi_{2}(x, \lambda), \cdots, \chi_{n}(x, \lambda)$ of the equation (1.1) on whole $J=$ $[-1,0) \cup(0,1]$.

Let $\phi_{11}(x, \lambda), \phi_{12}(x, \lambda), \cdots, \phi_{1 n}(x, \lambda)$ and $\chi_{11}(x, \lambda), \chi_{12}(x, \lambda), \cdots, \chi_{1 n}(x, \lambda)$ be solutions of the equation $(1.1)$ in the interval $[-1,0)$ satisfying the following initial conditions

$$
\begin{aligned}
& \left(C_{\phi_{11}}, C_{\phi_{12}}, \cdots, C_{\phi_{1 n}}, C_{\chi_{11}}, C_{\chi_{12}}, \cdots, C_{\chi_{1 n}}\right)(-1, \lambda) \\
= & \left(\begin{array}{cccccccc}
a_{2 n} & 0 & \cdots & 0 & 0 & \cdots & 0 & 0 \\
0 & a_{2 n-1} & \cdots & 0 & 0 & \cdots & 0 & 0 \\
\cdots & \cdots & \cdots & \cdots & \cdots & \cdots & \cdots & \cdots \\
0 & 0 & \cdots & a_{n+1} & 0 & \cdots & 0 & 0 \\
0 & 0 & \cdots & -a_{n} & 1 & \cdots & 0 & 0 \\
0 & -a_{2} & \cdots & 0 & 0 & \cdots & 1 & 0 \\
-a_{1} & 0 & \cdots & 0 & 0 & \cdots & 0 & 1
\end{array}\right) .
\end{aligned}
$$

As same as above, we let $\phi_{21}(x, \lambda), \phi_{22}(x, \lambda), \cdots, \phi_{2 n}(x, \lambda)$ and $\chi_{21}(x, \lambda)$, $\chi_{22}(x, \lambda), \cdots, \chi_{2 n}(x, \lambda)$ be solutions of the equation (1.1) satisfying the following initial conditions

$$
\begin{aligned}
& \left.C_{\phi_{21}}, C_{\phi_{22}}, \cdots, C_{\phi_{2 n}}, C_{\chi_{21}}, C_{\chi_{22}}, \cdots, C_{\chi_{2 n}}\right)(0, \lambda) \\
= & C \cdot\left(C_{\phi_{11}}, C_{\phi_{12}}, \cdots, C_{\phi_{1 n}}, C_{\chi_{11}}, C_{\chi_{12}}, \cdots, C_{\chi_{1 n}}\right)(0, \lambda) .
\end{aligned}
$$

The Wronskian $w\left(\phi_{i 1}, \cdots, \phi_{i n}, \chi_{i 1}, \cdots, \chi_{i n}\right)(x, \lambda)(i=1,2)$ are independent of the variable $x$, and are entire functions of parameter $\lambda$.

Let

$$
w_{i}(\lambda)=w\left(\phi_{i 1}, \cdots, \phi_{i n}, \chi_{i 1}, \cdots, \chi_{i n}\right)(x, \lambda)(i=1,2) .
$$

Since $w_{i}(\lambda)$ are independent of the variable $x$, by (3.1), (3.2), we have

$$
\begin{aligned}
w_{1}(\lambda) & =w\left(\phi_{11}, \phi_{12}, \cdots, \phi_{1 n}, \chi_{11}, \chi_{12}, \cdots, \chi_{1 n}\right)(x, \lambda) \\
& =\operatorname{det}\left(C_{\phi_{11}}, C_{\phi_{12}}, \cdots, C_{\phi_{1 n}}, C_{\chi_{11}}, C_{\chi_{12}}, \cdots, C_{\chi_{1 n}}\right)(x, \lambda) \\
& =\operatorname{det}\left(C_{\phi_{11}}, C_{\phi_{12}}, \cdots, C_{\phi_{1 n}}, C_{\chi_{11}}, C_{\chi_{12}}, \cdots, C_{\chi_{1 n}}\right)(-1, \lambda) \\
& =a_{n+1} a_{n+2} \cdots a_{2 n} \neq 0,
\end{aligned}
$$


and

$$
\begin{aligned}
w_{2}(\lambda) & =w\left(\phi_{21}, \phi_{22}, \cdots, \phi_{2 n}, \chi_{21}, \chi_{22}, \cdots, \chi_{2 n}\right)(x, \lambda) \\
& =\operatorname{det}\left(C_{\phi_{21}}, C_{\phi_{22}}, \cdots, C_{\phi_{2 n}}, C_{\chi_{21}}, C_{\chi_{22}}, \cdots, C_{\chi_{2 n}}\right)(x, \lambda) \\
& =\operatorname{det}\left(C_{\phi_{21}}, C_{\phi_{22}}, \cdots, C_{\phi_{2 n}}, C_{\chi_{21}}, C_{\chi_{22}}, \cdots, C_{\chi_{2 n}}\right)(0, \lambda) \\
& =\operatorname{det}\left(C \cdot\left(C_{\phi_{11}}, C_{\phi_{12}}, \cdots, C_{\phi_{1 n}}, C_{\chi_{11}}, C_{\chi_{12}}, \cdots, C_{\chi_{1 n}}\right)(0, \lambda)\right) \\
& =(\operatorname{det} C) w_{1}(\lambda)=\rho^{n} w_{1}(\lambda) \neq 0 .
\end{aligned}
$$

So the functions $\phi_{21}(x, \lambda), \phi_{22}(x, \lambda), \cdots, \phi_{2 n}(x, \lambda)$ and $\chi_{21}(x, \lambda), \chi_{22}(x, \lambda)$, $\cdots, \chi_{2 n}(x, \lambda)$ are linearly independent in the interval $(0,1]$.

Let

$$
\begin{array}{r}
\phi_{1}(x, \lambda)=\left\{\begin{array}{ll}
\phi_{11}(x, \lambda), & x \in[-1,0), \\
\phi_{21}(x, \lambda), & x \in(0,1] ;
\end{array}, \phi_{2}(x, \lambda)= \begin{cases}\phi_{12}(x, \lambda), & x \in[-1,0), \\
\phi_{22}(x, \lambda), & x \in(0,1] ;\end{cases} \right. \\
\cdots \cdots, \phi_{n}(x, \lambda)= \begin{cases}\phi_{1 n}(x, \lambda), & x \in[-1,0), \\
\phi_{2 n}(x, \lambda), & x \in(0,1] ;\end{cases}
\end{array}
$$

and

$$
\begin{array}{r}
\chi_{1}(x, \lambda)=\left\{\begin{array}{ll}
\chi_{11}(x, \lambda), & x \in[-1,0), \\
\chi_{21}(x, \lambda), & x \in(0,1] ;
\end{array}, \chi_{2}(x, \lambda)=\left\{\begin{array}{ll}
\chi_{12}(x, \lambda), & x \in[-1,0), \\
\chi_{22}(x, \lambda), & x \in(0,1] ;
\end{array},\right.\right. \\
\cdots \cdots, \chi_{n}(x, \lambda)= \begin{cases}\chi_{1 n}(x, \lambda), & x \in[-1,0) ; \\
\chi_{2 n}(x, \lambda), & x \in(0,1] .\end{cases}
\end{array}
$$

Moreover, $\phi_{1}(x, \lambda), \phi_{2}(x, \lambda), \cdots, \phi_{n}(x, \lambda)$ and $\chi_{1}(x, \lambda), \chi_{2}(x, \lambda), \cdots, \chi_{n}(x, \lambda)$ satisfy the boundary conditions (1.2) and transmission conditions (1.4). They are independent of $x$, and are entire functions of parameter $\lambda$.

Lemma 4. The following determinant is equal to $w_{1}(\lambda)$, or $-w_{1}(\lambda)$, i.e.

$$
\left|\begin{array}{cccc}
l_{1} \chi_{11} & l_{1} \chi_{12} & \cdots & l_{1} \chi_{1 n} \\
l_{2} \chi_{11} & l_{2} \chi_{12} & \cdots & l_{2} \chi_{1 n} \\
\cdots & \cdots & \cdots & \cdots \\
l_{n} \chi_{11} & l_{n} \chi_{12} & \cdots & l_{n} \chi_{1 n}
\end{array}\right|=w_{1}(\lambda), \text { or }=-w_{1}(\lambda)
$$

if $n=1,4,5,8,9, \cdots$, then the determinant is equal to $w_{1}(\lambda)$;

if $n=2,3,6,7, \cdots$, then it is equal to $-w_{1}(\lambda)$.

Where

$$
\begin{gathered}
l_{1} \chi_{1 i}=a_{1} \chi_{1 i}(-1, \lambda)+a_{2 n} \chi_{1 i}^{(2 n-1)}(-1, \lambda), i=1,2, \cdots, n, \\
l_{2} \chi_{1 i}=a_{2} \chi_{1 i}^{\prime}(-1, \lambda)+a_{2 n-1} \chi_{1 i}^{(2 n-2)}(-1, \lambda), i=1,2, \cdots, n, \\
\cdots \cdots \cdots \\
l_{n} \chi_{1 i}=a_{n} \chi_{1 i}^{(n-1)}(-1, \lambda)+a_{n+1} \chi_{1 i}^{(n)}(-1, \lambda), i=1,2, \cdots, n .
\end{gathered}
$$


Proof. By (3.1), we have

$$
\begin{aligned}
& \left|\begin{array}{cccc}
l_{1} \chi_{11} & l_{1} \chi_{12} & \cdots & l_{1} \chi_{1 n} \\
l_{2} \chi_{11} & l_{2} \chi_{12} & \cdots & l_{2} \chi_{1 n} \\
\cdots & \cdots & \cdots & \cdots \\
l_{n} \chi_{11} & l_{n} \chi_{12} & \cdots & l_{n} \chi_{1 n}
\end{array}\right|= \\
& \left|\begin{array}{cccc}
a_{1} \chi_{11}+a_{2 n} \chi_{11}^{(2 n-1)} & a_{1} \chi_{12}+a_{2 n} \chi_{12}^{(2 n-1)} & \cdots & a_{1} \chi_{1 n}+a_{2 n} \chi_{1 n}^{(2 n-1)} \\
a_{2} \chi_{11}^{\prime}+a_{2 n-1} \chi_{11}^{(2 n-2)} & a_{2} \chi_{12}^{\prime}+a_{2 n-1} \chi_{12}^{(2 n-2)} & \cdots & a_{2} \chi_{1 n}^{\prime}+a_{2 n-1} \chi_{1 n}^{(2 n-2)} \\
\cdots & \cdots & \cdots & \cdots \\
a_{n} \chi_{11}^{(n-1)}+a_{n+1} \chi_{11}^{(n)} & a_{n} \chi_{12}^{(n-1)}+a_{n+1} \chi_{12}^{(n)} & \cdots & a_{n} \chi_{1 n}^{(n-1)}+a_{n+1} \chi_{1 n}^{(n)}
\end{array}\right| \\
& =\left|\begin{array}{cccc}
0 & \cdots & 0 & a_{2 n} \\
0 & \cdots & a_{2 n-1} & 0 \\
\cdots & \cdots & \cdots & \cdots \\
a_{n+1} & \cdots & 0 & 0
\end{array}\right|=a_{n+1} a_{n+2} \cdots a_{2 n}, \text { or }=-a_{n+1} a_{n+2} \cdots a_{2 n},
\end{aligned}
$$

if $n=1,4,5,8,9, \cdots$, then it is equal to $a_{n+1} a_{n+2} \cdots a_{2 n}$, i.e. $w_{1}(\lambda)$; if $n=2,3,6,7, \cdots$, then it is equal to $-a_{n+1} a_{n+2} \cdots a_{2 n}$, i.e. $-w_{1}(\lambda)$. We complete the proof.

Lemma 5. Let

$$
u(x)= \begin{cases}u_{1}(x), & x \in[-1,0), \\ u_{2}(x), & x \in(0,1]\end{cases}
$$

be any solution of the equation ly $=\lambda y$, then it can be represented as

$$
u(x)= \begin{cases}d_{1} \phi_{11}+\cdots+d_{n} \phi_{1 n}+d_{n+1} \chi_{11}+\cdots+d_{2 n} \chi_{1 n}, & x \in[-1,0), \\ d_{2 n+1} \phi_{21}+\cdots+d_{3 n} \phi_{2 n}+d_{3 n+1} \chi_{21}+\cdots+d_{4 n} \chi_{2 n}, & x \in(0,1] ;\end{cases}
$$

where $d_{i} \in \mathbb{C}(i=1,2, \cdots, 4 n)$. If $u(x)$ satisfies the transmission conditions (1.4), then $d_{1}=d_{2 n+1}, d_{2}=d_{2 n+2}, \cdots, d_{2 n}=d_{4 n}$.

Proof. Let $u(x)$ is represented in the form

$$
u(x)=\left\{\begin{array}{ll}
d_{1} \phi_{11}+\cdots+d_{n} \phi_{1 n}+d_{n+1} \chi_{11}+\cdots+d_{2 n} \chi_{1 n}, & x \in[-1,0), \\
d_{2 n+1} \phi_{21}+\cdots+d_{3 n} \phi_{2 n}+d_{3 n+1} \chi_{21}+\cdots+d_{4 n} \chi_{2 n}, & x \in(0,1] ;
\end{array} .\right.
$$

Apply the transmission conditions (1.4) to this representation of $u(x)$. Since

$$
u^{(i-1)}(0+)=\sum_{j=1}^{2 n} c_{i j} u^{(j-1)}(0-), i=1,2, \cdots, 2 n,
$$

i.e.

$$
C_{u}(0+)=C \cdot C_{u}(0-)
$$




$$
\begin{gathered}
\left(\begin{array}{c}
d_{2 n+1} \phi_{21}+\cdots+d_{3 n} \phi_{2 n}+d_{3 n+1} \chi_{21}+\cdots+d_{4 n} \chi_{2 n} \\
d_{2 n+1} \phi_{21}^{\prime}+\cdots+d_{3 n} \phi_{2 n}^{\prime}+d_{3 n+1} \chi_{21}^{\prime}+\cdots+d_{4 n} \chi_{2 n}^{\prime} \\
\cdots \\
d_{2 n+1} \phi_{21}^{(2 n-1)}+\cdots+d_{3 n} \phi_{2 n}^{(2 n-1)}+d_{3 n+1} \chi_{21}^{(2 n-1)}+\cdots+d_{4 n} \chi_{2 n}^{(2 n-1)}
\end{array}\right)(0, \lambda) \\
=C\left(\begin{array}{c}
d_{1} \phi_{11}+\cdots+d_{n} \phi_{1 n}+d_{n+1} \chi_{11}+\cdots+d_{2 n} \chi_{1 n} \\
d_{1} \phi_{11}^{\prime}+\cdots+d_{n} \phi_{1 n}^{\prime}+d_{n+1} \chi_{11}^{\prime}+\cdots+d_{2 n} \chi_{1 n}^{\prime} \\
\cdots \\
d_{1} \phi_{11}^{(2 n-1)}+\cdots+d_{n} \phi_{1 n}^{(2 n-1)}+d_{n+1} \chi_{11}^{(2 n-1)}+\cdots+d_{2 n} \chi_{1 n}^{(2 n-1)}
\end{array}\right)(0, \lambda),
\end{gathered}
$$

we rewrite it in the following form

$$
\begin{array}{r}
\left(C_{\phi_{21}}, C_{\phi_{22}}, \cdots, C_{\phi_{2 n}}, C_{\chi_{21}}, C_{\chi_{22}}, \cdots, C_{\chi_{2 n}}\right)(0, \lambda)\left(\begin{array}{c}
d_{2 n+1} \\
d_{2 n+2} \\
\cdots \\
d_{4 n}
\end{array}\right) \\
=C \cdot\left(C_{\phi_{11}}, C_{\phi_{12}}, \cdots, C_{\phi_{1 n}}, C_{\chi_{11}}, C_{\chi_{12}}, \cdots, C_{\chi_{1 n}}\right)(0, \lambda)\left(\begin{array}{c}
d_{1} \\
d_{2} \\
\cdots \\
d_{2 n}
\end{array}\right),
\end{array}
$$

taking into account the initial conditions (3.2) for $\phi_{21}(x, \lambda), \cdots, \phi_{2 n}(x, \lambda)$ and $\chi_{21}(x, \lambda), \cdots, \chi_{2 n}(x, \lambda)$, we have

$$
\begin{gathered}
C \cdot\left(C_{\phi_{11}}, C_{\phi_{12}}, \cdots, C_{\phi_{1 n}}, C_{\chi_{11}}, C_{\chi_{12}}, \cdots, C_{\chi_{1 n}}\right)(0, \lambda)\left(\begin{array}{c}
d_{2 n+1} \\
d_{2 n+2} \\
\cdots \\
d_{4 n}
\end{array}\right) \\
=C \cdot\left(C_{\phi_{11}}, C_{\phi_{12}}, \cdots, C_{\phi_{1 n}}, C_{\chi_{11}}, C_{\chi_{12}}, \cdots, C_{\chi_{1 n}}\right)(0, \lambda)\left(\begin{array}{c}
d_{1} \\
d_{2} \\
\cdots \\
d_{2 n}
\end{array}\right) .
\end{gathered}
$$

So

$$
C \cdot\left(C_{\phi_{11}}, C_{\phi_{12}}, \cdots, C_{\phi_{1 n}}, C_{\chi_{11}}, C_{\chi_{12}}, \cdots, C_{\chi_{1 n}}\right)(0, \lambda)\left(\begin{array}{c}
d_{2 n+1}-d_{1} \\
d_{2 n+2}-d_{2} \\
\cdots \\
d_{4 n}-d_{2 n}
\end{array}\right)=0 .
$$

Since

$$
\operatorname{det}\left(C\left(C_{\phi_{11}}, C_{\phi_{12}}, \cdots, C_{\phi_{1 n}}, C_{\chi_{11}}, C_{\chi_{12}}, \cdots, C_{\chi_{1 n}}\right)(0, \lambda)\right)=\rho^{n} w_{1}(\lambda) \neq 0,
$$

the above equation (3.5) has only zero solution, $d_{1}=d_{2 n+1}, d_{2}=d_{2 n+2}$, $\cdots, d_{2 n}=d_{4 n}$. 
For convenience, we let

$$
B_{\lambda}=\left(\begin{array}{cccccc}
\lambda b_{1}^{\prime}+b_{1} & \cdots & 0 & 0 & \cdots & \lambda b_{2 n}^{\prime}+b_{2 n} \\
0 & \cdots & 0 & 0 & \cdots & 0 \\
\cdots & \cdots & \cdots & \cdots & \cdots & \cdots \\
0 & \cdots & \lambda b_{n}^{\prime}+b_{n} & \lambda b_{n+1}^{\prime}+b_{n+1} & \cdots & 0
\end{array}\right)
$$

and

SO

$$
\operatorname{det} \Phi(1, \lambda)=\left|\begin{array}{cccc}
l_{n+1} \phi_{21} & l_{n+1} \phi_{22} & \cdots & l_{n+1} \phi_{2 n} \\
l_{n+2} \phi_{21} & l_{n+2} \phi_{22} & \cdots & l_{n+2} \phi_{2 n} \\
\cdots & \cdots & \cdots & \cdots \\
l_{2 n} \phi_{21} & l_{2 n} \phi_{22} & \cdots & l_{2 n} \phi_{2 n}
\end{array}\right|
$$

$$
\operatorname{det} \Phi(1, \lambda)=\operatorname{det}\left(B_{\lambda} \cdot\left(C_{\phi_{21}}, C_{\phi_{22}}, \cdots, C_{\phi_{2 n}}\right)(1, \lambda)\right),
$$

where

$$
\begin{gathered}
l_{n+1} \phi_{2 i}=\left(\lambda b_{1}^{\prime}+b_{1}\right) \phi_{2 i}(1, \lambda)+\left(\lambda b_{2 n}^{\prime}+b_{2 n}\right) \phi_{2 i}^{(2 n-1)}(1, \lambda), i=1,2, \cdots, n, \\
l_{n+2} \phi_{2 i}=\left(\lambda b_{2}^{\prime}+b_{2}\right) \phi_{2 i}^{\prime}(1, \lambda)+\left(\lambda b_{2 n-1}^{\prime}+b_{2 n-1}\right) \phi_{2 i}^{(2 n-2)}(1, \lambda), i=1,2, \cdots, n, \\
\ldots \ldots \ldots \\
l_{2 n} \phi_{2 i}=\left(\lambda b_{n}^{\prime}+b_{n}\right) \phi_{2 i}^{(n-1)}(1, \lambda)+\left(\lambda b_{n+1}^{\prime}+b_{n+1}\right) \phi_{2 i}^{(n)}(1, \lambda), i=1,2, \cdots, n .
\end{gathered}
$$

Theorem 2. The eigenvalues of the problem (1.1)-(1.4) coincide with the zeros of the entire function $\operatorname{det} \Phi(1, \lambda)$.

Proof. Let $\lambda_{0}$ be an eigenvalue and $u_{0}(x)$ be any corresponding eigenfunction. Show that $\operatorname{det} \Phi\left(1, \lambda_{0}\right)=0$. If not, $\operatorname{det} \Phi\left(1, \lambda_{0}\right) \neq 0$. From (3.3) and (3.4) we know that $w_{1}\left(\lambda_{0}\right) \neq 0$ and $w_{2}\left(\lambda_{0}\right) \neq 0$. Consequently, each group of functions $\phi_{11}\left(x, \lambda_{0}\right), \cdots, \phi_{1 n}\left(x, \lambda_{0}\right), \chi_{11}\left(x, \lambda_{0}\right), \cdots, \chi_{1 n}\left(x, \lambda_{0}\right)$ and $\phi_{21}\left(x, \lambda_{0}\right), \cdots$, $\phi_{2 n}\left(x, \lambda_{0}\right), \chi_{21}\left(x, \lambda_{0}\right), \cdots, \chi_{2 n}\left(x, \lambda_{0}\right)$ would be linearly independent on $[-1,0)$ and $(0,1]$, respectively. Therefore eigenfunction $u_{0}(x)$ might be represented in the form

$$
u_{0}(x)= \begin{cases}\left(d_{1} \phi_{11}+\cdots+d_{n} \phi_{1 n}+d_{n+1} \chi_{11}\right. & \\ \left.+\cdots+d_{2 n} \chi_{1 n}\right)\left(x, \lambda_{0}\right), & x \in[-1,0) \\ \left(d_{2 n+1} \phi_{21}+\cdots+d_{3 n} \phi_{2 n}+d_{3 n+1} \chi_{11}\right. & \\ \left.+\cdots+d_{4 n} \chi_{2 n}\right)\left(x, \lambda_{0}\right), & x \in(0,1]\end{cases}
$$

where at least one of the constants $d_{1}, d_{2}, \cdots, d_{4 n}$ is not zero. By substituting this representation in the conditions (1.2)-(1.4), we obtain a system of linear, homogeneous equations for the variables of the constants $d_{1}, d_{2}, \cdots, d_{4 n}$. The considered equations are

$$
l_{k}\left(u_{0}(x)\right)=0, k=1,2, \cdots, 4 n .
$$


In fact

$$
\begin{gathered}
l_{i} u_{0}=\sum_{k=1}^{n} d_{n+k}\left(a_{i} \chi_{1 k}^{(i-1)}(-1)+a_{2 n+1-i} \chi_{1 k}^{(2 n-i)}(-1)\right)=0, i=1,2, \cdots, n, \\
l_{n+i} u_{0}=\sum_{k=1}^{n} d_{2 n+k}\left(\left(\lambda_{0} b_{i}^{\prime}+b_{i}\right) \phi_{2 k}^{(i-1)}(1)+\left(\lambda_{0} b_{2 n+1-i}^{\prime}+b_{2 n+1-i}\right) \phi_{2 k}^{(2 n-i)}(1)\right) \\
+\sum_{k=1}^{n} d_{3 n+k}\left(\left(\lambda_{0} b_{i}^{\prime}+b_{i}\right) \chi_{2 k}^{(i-1)}(1)+\left(\lambda_{0} b_{2 n+1-i}^{\prime}+b_{2 n+1-i}\right) \chi_{2 k}^{(2 n-i)}(1)\right)=0, i=1,2, \cdots, n, \\
l_{2 n+i} u_{0}=-\sum_{k=1}^{n} d_{k} \phi_{2 k}^{(i-1)}(0)-\sum_{k=1}^{n} d_{n+k} \chi_{2 k}^{(i-1)}(0)+\sum_{k=1}^{n} d_{2 n+k} \phi_{2 k}^{(i-1)}(0) \\
+\sum_{k=1}^{n} d_{3 n+k} \chi_{2 k}^{(i-1)}(0), i=1,2, \cdots, 2 n .
\end{gathered}
$$

By Lemma 4, we obtain that the determinant of this system is equal to

$$
(-1)^{n^{2}} w_{1}\left(\lambda_{0}\right) w_{2}\left(\lambda_{0}\right) \operatorname{det} \Phi\left(1, \lambda_{0}\right) \neq 0,
$$

or is equal to

$$
(-1)^{n^{2}+1} w_{1}\left(\lambda_{0}\right) w_{2}\left(\lambda_{0}\right) \operatorname{det} \Phi\left(1, \lambda_{0}\right) \neq 0,
$$

where

$$
\begin{gathered}
l_{n+1} \chi_{2 i}=\left(\lambda b_{1}^{\prime}+b_{1}\right) \chi_{2 i}(1, \lambda)+\left(\lambda b_{2 n}^{\prime}+b_{2 n}\right) \chi_{2 i}^{(2 n-1)}(1, \lambda), i=1,2, \cdots, n, \\
l_{n+2} \chi_{2 i}=\left(\lambda b_{2}^{\prime}+b_{2}\right) \chi_{2 i}^{\prime}(1, \lambda)+\left(\lambda b_{2 n-1}^{\prime}+b_{2 n-1}\right) \chi_{2 i}^{(2 n-2)}(1, \lambda), i=1,2, \cdots, n, \\
\cdots \cdots \cdots \\
l_{2 n} \chi_{2 i}=\left(\lambda b_{n}^{\prime}+b_{n}\right) \chi_{2 i}^{(n-1)}(1, \lambda)+\left(\lambda b_{n+1}^{\prime}+b_{n+1}\right) \chi_{2 i}^{(n)}(1, \lambda), i=1,2, \cdots, n, \\
\phi_{2 k}^{(i-1)}=\phi_{2 k}^{(i-1)}(0), \chi_{2 k}^{(i-1)}=\chi_{2 k}^{(i-1)}(0), k=1,2, \cdots, n, i=1,2, \cdots, 2 n .
\end{gathered}
$$

Therefore, the system (3.6) has the only trivial solution $d_{1}=d_{2}=\cdots=d_{4 n}=0$. Thus, we get the contradiction.

On the contrary, if $\operatorname{det} \Phi\left(1, \lambda_{0}\right)=0$, the homogeneous equations for the variables of the constants $c_{1}, c_{2}, \cdots, c_{2 n}$,

$$
\Phi(1, \lambda)\left(\begin{array}{c}
c_{1} \\
c_{2} \\
\cdots \\
c_{2 n}
\end{array}\right)=0
$$


has nonzero solution $\left(c_{1}^{\prime}, c_{2}^{\prime}, \cdots, c_{2 n}^{\prime}\right)^{T}$. By Lemma 5 , let

$$
y(x)= \begin{cases}\left(c_{1}^{\prime} \phi_{11}+\cdots+c_{n}^{\prime} \phi_{1 n}+c_{n+1}^{\prime} \chi_{11}+\cdots+c_{2 n}^{\prime} \chi_{1 n}\right)(x, \lambda), & x \in(-1,0), \\ \left(c_{1}^{\prime} \phi_{21}+\cdots+c_{n}^{\prime} \phi_{2 n}+c_{n+1}^{\prime} \chi_{21}+\cdots+c_{2 n}^{\prime} \chi_{2 n}\right)(x, \lambda), & x \in(0,1] ;\end{cases}
$$

then $y(x)$ is the nonzero solution of equation $l(y)=\lambda y$, which satisfy conditions (1.3)-(1.4). so $\lambda$ is the eigenvalue of the problem (1.1)-(1.4). Which completes the proof.

Corollary 3. The eigenvalues of the problem (1.1)-(1.4) are real and form a finite or infinite sequence without finite accumulation point.

\section{Completeness of Eigenfunctions}

In this section, in terms of new Hilbert space $H$ and new operator $A$, we investigate the problem (1.1)-(1.4), and obtain the following conclusion.

Theorem 3. The operator $A$ has only point spectrum, i.e. $\sigma(A)=\sigma_{p}(A)$.

Proof. Let $\gamma \bar{\epsilon} \sigma_{p}(A)$. We only show $\gamma \in \rho(A)$. Since $A$ is self-adjoint, we only consider a real $\gamma$. We investigate the equation $(A-\gamma) Y=F$, where $F=$ $\left(f, h_{1}, \cdots, h_{n}\right) \in H, \gamma \in \mathbb{R}$.

Consider the following problem

$$
\left\{\begin{array}{l}
l y-\gamma y=f, x \in[-1,0) \cup(0,1] \\
l_{i} y=a_{i} y^{(i-1)}(-1)+a_{2 n+1-i} y^{(2 n-i)}(-1)=0, i=1,2, \cdots, n, \\
l_{2 n+i} y=y^{(i-1)}(0+)-\sum_{j=1}^{2 n} c_{i j} y^{(j-1)}(0-)=0, i=1,2, \cdots, 2 n
\end{array}\right.
$$

and equations

$$
M_{i}(y)+\gamma M_{i}^{\prime}(y)=-h_{i}, i=1,2, \cdots, n .
$$

Let $u(x)$ be the solution of the equation $l u-\gamma u=0$, satisfying

$$
\begin{gathered}
u^{(i-1)}(-1)=a_{2 n+1-i}, u^{(2 n-i)}(-1)=-a_{i}, i=1,2, \cdots, n, \\
u^{(i-1)}(0+)=\sum_{j=1}^{2 n} c_{i j} u^{(j-1)}(0-), i=1,2, \cdots, 2 n .
\end{gathered}
$$

In fact

$$
u(x)=\left\{\begin{array}{ll}
u_{1}(x), & x \in[-1,0), \\
u_{2}(x), & x \in(0,1] ;
\end{array},\right.
$$

where $u_{1}(x)$ is the unique solution of the initial-value problem

$$
\left\{\begin{array}{l}
-p_{1}^{2} u^{(2 n)}(x)+q(x) u(x)=\gamma u(x), x \in[-1,0), \\
u^{(i-1)}(-1)=a_{2 n+1-i}, u^{(2 n-i)}(-1)=-a_{i}, i=1,2, \cdots, n
\end{array},\right.
$$


and $u_{2}(x)$ is the unique solution of the initial-value problem

$$
\left\{\begin{array}{l}
-p_{2}^{2} u^{(2 n)}(x)+q(x) u(x)=\gamma u(x), x \in(0,1], \\
u^{(i-1)}(0+)=\sum_{j=1}^{2 n} c_{i j} u^{(j-1)}(0-), i=1,2, \cdots, 2 n ;
\end{array}\right.
$$

Let

$$
w(x)= \begin{cases}w_{1}(x), & x \in[-1,0), \\ w_{2}(x), & x \in(0,1] ;\end{cases}
$$

be a special solution of $l w-\gamma w=f$ satisfying

$$
\left\{\begin{array}{l}
a_{i} w^{(i-1)}(-1)+a_{2 n+1-i} w^{(2 n-i)}(-1)=0, \\
w^{(i-1)}(0+)=\sum_{j=1}^{2 n} c_{i j} w^{(j-1)}(0-), i=1,2, \cdots, 2 n ;
\end{array}\right.
$$

By Lemma 5, Equation (4.1) has the general solution in the form

$$
y(x)= \begin{cases}\left(d_{1} \phi_{11}+\cdots+d_{n} \phi_{1 n}+d_{n+1} \chi_{11}\right. & \\ \left.+\cdots+d_{2 n} \chi_{1 n}\right)(x, \gamma)+w_{1}, & x \in[-1,0), \\ \left(d_{1} \phi_{21}+\cdots+d_{n} \phi_{2 n}+d_{n+1} \chi_{21}\right. & \\ \left.+\cdots+d_{2 n} \chi_{2 n}\right)(x, \gamma)+w_{2}, & x \in(0,1] ;\end{cases}
$$

where $d_{i} \in \mathbb{C}(i=1,2, \cdots, 2 n)$.

Since $\gamma$ is not eigenvalue of the problem (1.1)-(1.4), at least one of the following expressions is not zero

$$
\begin{array}{r}
\gamma\left(b_{i}^{\prime} u^{(i-1)}(1)+b_{2 n+1-i}^{\prime} u^{(2 n-i)}(1)\right)+b_{i} u^{(i-1)}(1)+b_{2 n+1-i} u^{(2 n-i)}(1), \\
i=1,2, \cdots, n .
\end{array}
$$

The second, third, $\cdots, n$th components of the equation $(A-\gamma) Y=F$ involves the equation (4.2), i.e.

$$
\begin{array}{r}
\left(b_{i} y^{(i-1)}(1)+b_{2 n+1-i} y^{(2 n-i)}(1)\right)+\gamma\left(b_{i}^{\prime} y^{(i-1)}(1)\right. \\
\left.+b_{2 n+1-i}^{\prime} y^{(2 n-i)}(1)\right)=-h_{i}, i=1,2, \cdots, n .
\end{array}
$$

Substituting (4.3) into (4.5), we get

$$
\begin{aligned}
& \left(b_{i} \phi_{21}^{(i-1)}(1)+b_{2 n+1-i} \phi_{21}^{(2 n-i)}(1)+\gamma\left(b_{i}^{\prime} \phi_{21}^{(i-1)}(1)+b_{2 n+1-i}^{\prime} \phi_{21}^{(2 n-i)}(1)\right)\right) d_{1} \\
& +\left(b_{i} \phi_{22}^{(i-1)}(1)+b_{2 n+1-i} \phi_{22}^{(2 n-i)}(1)+\gamma\left(b_{i}^{\prime} \phi_{22}^{(i-1)}(1)+b_{2 n+1-i}^{\prime} \phi_{22}^{(2 n-i)}(1)\right)\right) d_{2} \\
+ & \cdots+\left(b_{i} \phi_{2 n}^{(i-1)}(1)+b_{2 n+1-i} \phi_{2 n}^{(2 n-i)}(1)+\gamma\left(b_{i}^{\prime} \phi_{2 n}^{(i-1)}(1)+b_{2 n+1-i}^{\prime} \phi_{2 n}^{(2 n-i)}(1)\right)\right) d_{n} \\
+ & \left(b_{i} \chi_{21}^{(i-1)}(1)+b_{2 n+1-i} \chi_{21}^{(2 n-i)}(1)+\gamma\left(b_{i}^{\prime} \chi_{21}^{(i-1)}(1)+b_{2 n+1-i}^{\prime} \chi_{21}^{(2 n-i)}(1)\right)\right) d_{n+1} \\
+ & \left(b_{i} \chi_{22}^{(i-1)}(1)+b_{2 n+1-i} \chi_{22}^{(2 n-i)}(1)+\gamma\left(b_{i}^{\prime} \chi_{22}^{(i-1)}(1)+b_{2 n+1-i}^{\prime} \chi_{22}^{(2 n-i)}(1)\right)\right) d_{n+2}
\end{aligned}
$$




$$
\begin{gathered}
+\cdots+\left(b_{i} \chi_{2 n}^{(i-1)}(1)+b_{2 n+1-i} \chi_{2 n}^{(2 n-i)}(1)+\gamma\left(b_{i}^{\prime} \chi_{21}^{(i-1)}(1)+b_{2 n+1-i}^{\prime} \chi_{2 n}^{(2 n-i)}(1)\right)\right) d_{2 n} \\
=-h_{i}-\left(b_{i} w_{2}^{(i-1)}(1)+b_{2 n+1-i} w_{2}^{(2 n-i)}(1)\right)-\gamma\left(b_{i}^{\prime} w_{2}(1)+b_{2 n+1-i}^{\prime} w_{2}^{(2 n-i)}(1)\right), \\
i=1,2, \cdots, n .
\end{gathered}
$$

We rewrite the above equations in following form, i.e.

$$
\begin{aligned}
& B_{\gamma}\left(C_{\phi_{21}}(1, \gamma), C_{\phi_{22}}(1, \gamma), \cdots, C_{\phi_{2 n}}(1, \gamma)\right)\left(\begin{array}{c}
d_{1} \\
d_{2} \\
\cdots \\
d_{2 n}
\end{array}\right) \\
= & \left(\begin{array}{c}
-h_{1} \\
-h_{2} \\
\cdots \\
-h_{n}
\end{array}\right)-B_{\gamma}\left(\begin{array}{c}
w_{2}(1) \\
w_{2}^{\prime}(1) \\
\cdots \\
w_{2}^{(2 n-1)}(1)
\end{array}\right) .
\end{aligned}
$$

The coefficient determinant of the equations about $d_{1}, d_{2}, \cdots, d_{2 n}$ is $\operatorname{det} \Phi(1, \gamma)$. Since $\gamma$ is not an eigenvalue, $\operatorname{det} \Phi(1, \gamma) \neq 0$, we know that $d_{1}, d_{2}, \cdots, d_{2 n}$ is uniquely solvable. So the general solution of the boundary value problem (4.1) is uniquely determined.

The above argument shows that $(A-\gamma I)^{-1}$ is defined on all of $H$. We obtain that $(A-\gamma I)^{-1}$ is bounded by Theorem 1 and by the closed Graph Theorem. Thus $\gamma \in \rho(A)$. Hence, $\sigma(A)=\sigma_{p}(A)$.

\section{REFERENCES}

[1] Z. Akdoğan, M. Demirci, and O. S. Mukhtarov, "Discontinuous Sturm-Liouville problems with eigenparameter-dependent boundary and transmissions conditions," Acta Appl. Math., vol. 86, no. 3, pp. 329-344, 2005.

[2] Z. Akdoğan, M. Demirci, and O. S. Mukhtarov, "Green function of discontinuous boundary-value problem with transmission conditions," Math. Methods Appl. Sci., vol. 30, no. 14, pp. 1719-1738, 2007.

[3] N. Altinisik, M. Kadakal, and O. S. Mukhtarov, "Eigenvalues and eigenfunctions of discontinuous Sturm-Liouville problems with eigenparameter-dependent boundary conditions," Acta Math. Hung., vol. 102, no. 1-2, pp. 159-175, 2004.

[4] P. A. Binding, P. J. Browne, and B. A. Watson, "Sturm-Liouville problems with boundary conditions rationally dependent on the eigenparameter. II," J. Comput. Appl. Math., vol. 148, no. 1, pp. 147-168, 2002.

[5] C. T. Fulton, "Two-point boundary value problems with eigenvalue parameter contained in the boundary conditions," Proc. R. Soc. Edinb., Sect. A, vol. 77, pp. 293-308, 1977.

[6] D. B. Hinton, "An expansion theorem for an eigenvalue problem with eigenvalue parameter in the boundary condition," Q. J. Math., Oxf. II. Ser., vol. 30, pp. 33-42, 1979.

[7] M. Kadakal and O. S. Mukhtarov, "Discontinuous Sturm-Liouville problems containing eigenparameter in the boundary conditions," Acta Math. Sin., Engl. Ser., vol. 22, no. 5, pp. 1519-1528, 2006. 
[8] M. Kadakal, O. S. Mukhtarov, and D. F. S. Muhtarov, "Some spectral properties of SturmLiouville problem with transmission conditions," Iran. J. Sci. Technol., Trans. A, Sci., vol. 29, no. 2, pp. 229-245, 2005.

[9] O. Mukhtarov, "Discontinuous boundary value problem with spectral parameter in boundary conditions," Turk. J. Math., vol. 18, no. 2, pp. 183-192, 1994.

[10] M. A. Naimark, Linear differential operators. Part II: Linear differential operators in Hilbert space. With additional material by the author. Translated by E. R. Dawson. English translation edited by W. N. Everitt. New York: Frederick Ungar Publishing Co., 1968.

[11] A. A. Shkalikov, "Boundary value problems for ordinary differential equations with a parameter in the boundary conditions," Tr. Semin. Im. I. G. Petrovskogo, vol. 9, pp. 190-229, 1983.

[12] I. Titeux and Y. Yakubov, "Completeness of root functions for thermal conduction in a strip with piecewise continuous coefficients," Math. Models Methods Appl. Sci., vol. 7, no. 7, pp. 1035-1050, 1997.

[13] A. Wang, J. Sun, X. Hao, and S. Yao, "Completeness of eigenfunctions of Sturm-Liouville problems with transmission conditions," Methods Appl. Anal., vol. 16, no. 3, pp. 299-312, 2009.

[14] A. Zettl, "Adjoint and self-adjoint boundary value problems with interface conditions," SIAM J. Appl. Math., vol. 16, pp. 851-859, 1968.

\section{Authors' addresses}

\section{Xin-yan Zhang}

School of Mathematical Sciences, Inner Mongolia University, 010021, Hohhot, China, and School of Statistics and Mathematics, Inner Mongolia University of Finance and Economics, 010070, Hohhot, China

E-mail address: zxinyan2006@163.com

\section{Jiong Sun}

School of Mathematical Sciences, Inner Mongolia University, 010021, Hohhot, China

E-mail address: masun@imu.edu.cn 